Epidemiology and Infection

cambridge.org/hyg

\section{Original Paper}

Cite this article: Zajaczkowski P, Mazumdar S, Conaty S, Ellis JT, Fletcher-Lartey SM (2019). Epidemiology and associated risk factors of giardiasis in a peri-urban setting in New South Wales Australia. Epidemiology and Infection 147, e15, 1-9. https://doi.org/10.1017/ S0950268818002637

Received: 14 March 2018

Revised: 29 August 2018

Accepted: 30 August 2018

\section{Key words:}

Control; diarrhoea; epidemiology; giardiasis; surveillance; transmission

Author for correspondence:

S. M. Fletcher-Lartey, E-mail: stephanie.

fletcher@health.nsw.gov.au

\title{
Epidemiology and associated risk factors of giardiasis in a peri-urban setting in New South Wales Australia
}

P. Zajaczkowski ${ }^{1}$, S. Mazumdar ${ }^{2}$, S. Conaty ${ }^{3}$, J. T. Ellis ${ }^{1}$ and S. M. Fletcher-Lartey ${ }^{3}$

${ }^{1}$ Faculty of Science, School of Life Sciences, University of Technology Sydney, Australia; ${ }^{2}$ Healthy People and Places Unit, South Western Sydney Local Health District, Liverpool, Australia and ${ }^{3}$ Public Health Unit, South Western Sydney Local Health District, Liverpool, Australia

\begin{abstract}
Giardiasis is one of the most important non-viral causes of human diarrhoea. Yet, little is known about the epidemiology of giardiasis in the context of developed countries such as Australia and there is a limited information about local sources of exposure to inform prevention strategies in New South Wales. This study aimed to (1) describe the epidemiology of giardiasis and (2) identify potential modifiable risk factors associated with giardiasis that are unique to south-western Sydney, Australia. A 1:2 matched case-control study of 190 confirmed giardiasis cases notified to the South-Western Local Health District Public Health Unit from January to December 2016 was employed to investigate the risk factors for giardiasis. Two groups of controls were selected to increase response rate; Pertussis cases and neighbourhood $(\mathrm{NBH})$ controls. A matched analysis was carried out for both control groups separately. Variables with a significant odds ratio (OR) in the univariate analysis were placed into a multivariable regression for each matched group, respectively. In the regression model with the NBH controls, age and sex were controlled as potential confounders. Identified risk factors included being under 5 years of age $(\mathrm{aOR}=7.08$; $95 \%$ confidence intervals $(\mathrm{CI}) 1.02$ $49.36)$, having a household member diagnosed with a gastrointestinal illness $(\mathrm{aOR}=15.89$; 95\% CI 1.53-164.60) and having contact with farm animals, domestic animals or wildlife $(\mathrm{aOR}=3.03$; 95\% CI 1.08-8.54). Cases that travelled overseas were at increased risk of infection $(\mathrm{aOR}=19.89 ; 95 \%$ CI 2.00-197.37) when compared with Pertussis cases. This study provides an update on the epidemiology and associated risk factors of a neglected tropical disease, which can inform enhanced surveillance and prevention strategies in the developed metropolitan areas.
\end{abstract}

\section{Introduction}

Giardia duodenalis (also known as Giardia lamblia or Giardia intestinalis) is one of the most common enteroparasites affecting humans with an estimated 280 million people being infected each year, around the world [1]. It is a protozoan parasite that causes infection in the bowel and clinically manifests as a diarrhoeal illness. Additionally, giardiasis has been associated with the development of chronic diarrhoea or irritable bowel syndrome, debilitating fatigue and reactive arthritis [2]. Giardiasis is not a life-threatening disease, however, infections may often go unnoticed due to many cases having a lack of symptoms. If left without treatment, the infection can become serious; impairing the development of children and resulting in a failure to thrive [3]. Certainly, giardiasis contributes negatively to public health development of endemic countries and causes devastating socio-economic loss. In 2004, G. duodenalis was officially included in the WHO Neglected Diseases Initiative [4]. Meanwhile, in Australia, giardiasis is a notifiable disease in several states and territories including New South Wales (NSW) [5].

Giardiasis is the most common notifiable parasitic infection in NSW. While the burden of disease is greater in developing settings with poor access to water, sanitation and hygiene (WASH) facilities, sporadic cases occur in developed countries including Australia and outbreaks are not uncommon [6]. In 2014, nearly 3000 cases were notified by laboratories in NSW [7] and 3434 cases reported in 2015 [7]. South Western Sydney (SWS) accounts for approximately $6 \%$ of cases state-wide. Amongst hospitalised patients, giardiasis was the second most commonly identified enteric protozoa, affecting mainly school age and young children [8]. In Australia, giardiasis is frequently associated with waterborne infections, day care centre disease outbreaks and travel-associated diarrhoea.

Few Australian studies have documented the prevalence of giardiasis; however, there are no recent studies that have examined the risk factors that drive local transmission of giardiasis $[9,10]$. The aim of this study was to describe the epidemiology of giardiasis and to identify the risk factors and sources of exposure associated with the disease in the SWS region of 
NSW. The study provides information on the impact of giardiasis on human health in SWS and a better understanding of the epidemiology and associated risk factors that can inform public health control strategies.

\section{Materials and methods}

\section{Study site}

The South-Western Sydney Local Health District (SWSLHD) was the research site. The SWSLHD includes seven Local Government Areas (LGA): Bankstown, Camden, Campbelltown, Fairfield, Liverpool, Wingecarribee and Wollondilly (see Supplementary Fig. S1).

The SWSLHD is the largest and fastest growing District in metropolitan Sydney. It has a large population of approximately 900000 , has a diverse geography, including significant populations in both rural and urban areas and approximately $46 \%$ of the population speak a language other than English at home. Public Health surveillance data can provide an example of what could be occurring across the NSW state.

\section{Study design and data collection}

\section{Case-control survey}

A 1:2 case-control study of risk factors was designed with the prospective recruitment of cases and controls. Cases were all confirmed cases of giardiasis notified to the SWSLHD Public Health Unit (PHU) from 1 January 2016 to 31 December 2016. A study questionnaire was developed based on a comprehensive review of the literature and was used to collect data from all cases and controls who agreed to participate in the study. Both case and control questionnaires are accessible online as Supplementary Material on the Cambridge Core website. The questionnaire asked about various socio-demographic features, self-reported clinical symptoms, information about care seeking behaviour and treatment received, the number of household members or other close contacts with similar symptoms and a range of exposures experienced 3 weeks before illness onset (for cases) or a similar time frame for controls. Enhanced data collection for this study also included additional details on potential confounders including country of birth, language spoken at home, highest educational attainment and occupation of the parents (for cases residing with their parents).

\section{Recruitment and selection of participants}

Laboratories are required under the NSW Public Health Act 2010 to notify PHUs of cases of giardiasis. As per the NSW Control Guideline protocols for investigation, once a giardiasis case was notified to the SWSLHD PHU, staff contacted the diagnosing doctor of the giardiasis case to request permission to contact the case or the parent or guardian (for persons under 16-years-old), to interview the case.

\section{Cases}

A 'case' was a person who had laboratory definitive evidence for the detection of G. duodenalis cysts or trophozoites in stool samples or samples of duodenal contents. Informed consent was provided by the case or their parent (for persons under 16 years); with parents/guardians asked to complete the responses on behalf of children 12-years-old or younger and to provide consent for children 13-15 years to answer their own questions.

\section{Controls}

A 'control' was defined as a person resident in SWSLHD and who did not have a history of a positive Giardia test in the previous 3 months (due to the possibility of chronic infection with Giardia). In order to improve the response rate and reduce selection bias, three different sets of controls were identified for the study.

(1) Control group 1: Neighbourhood controls (NBH):

Confirmed giardiasis cases were grouped into (i) urban and (ii) regional areas based on Australian Bureau of Standards regional classification. The aim was to identify 10 controls for each case to increase the likelihood of at least one household responding to the questionnaire. The following sampling strategy was employed.

(i) Urban: A list of all addresses in SWSLHD geocoded to latitude-longitude coordinates was obtained from the Geocoded National Address File. This dataset is available for free from 'Public Sector Mapping Agencies' Australia. A $500 \mathrm{~m}$ radius buffer (due to the dense population in urban areas) was drawn around each case's address using Geographic Information System tools (e.g. see Supplementary Fig. S2). Ten houses were then randomly selected from the list of addresses for each buffer.

(ii) Rural: The procedure followed was the same as for urban areas, except that $5 \mathrm{~km}$ buffers were used to account for population sparseness.

A letter with the Patient Information Statement and control questionnaire were sent to the selected household, with a request that the person with the next birthday in the household complete the questionnaire. The completed questionnaire was to be returned by post in the self-addressed envelope provided.

(2) Control group 2: Pertussis case:

Confirmed Pertussis cases notified in the same year, within the same age range ( \pm 5 years), residing within the same LGA but not on the same street as the corresponding giardiasis case were identified. If there were two or more persons meeting the criteria, one would be selected by simple random sampling using a random sampling function in Excel. Where no age match was available for the same LGA, one was selected from the closest LGA. Each control was contacted by telephone and once consent was obtained, the individual was interviewed with the standardised control questionnaire. If the person refused to participate in the study or was uncontactable after three phone calls, then the person was listed as a non-response.

(3) Control group 3: Friend Control:

This recruitment method yielded no controls and was not considered further.

\section{Sample size}

Based on surveillance data, it was estimated that the SWSLHD PHU received an average of 147 giardiasis notifications annually between the years 2012 and 2015. In a 1:3 unmatched design with a two-sided confidence level of $95 \%\left(z_{\alpha / 2}=1.96\right)$ with power $\left(z_{\beta}=0.80\right)$ of $80 \%$ and an estimated prevalence of a risk factor of $17 \%$ in controls and $40 \%$ in cases, at least 35 cases and 105 controls were needed to detect a significant risk of exposure (odds ratio $(\mathrm{OR})>3.25$ ) [11]. Oversampling of cases and controls was performed to accommodate for any non-responses or incompleteness in the data. As such, a total of 50 cases and 150 controls were needed. 

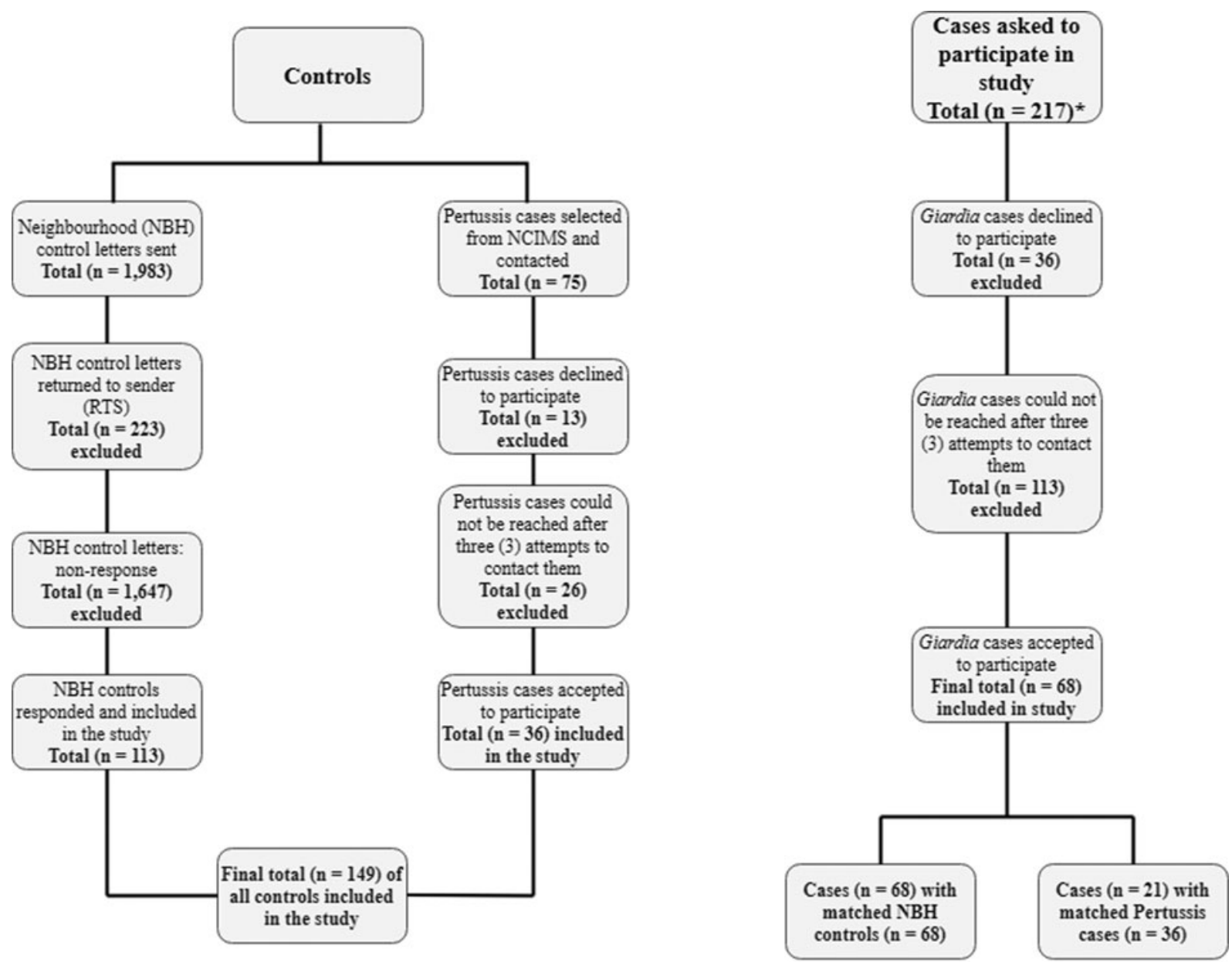

*including notified Giardia cases from January 2016 to January 2017

Fig. 1. A flowchart summary of the two different control types (neighbourhood control and Pertussis case) and the number of cases used in the study.

\section{Matched case-control analysis}

Survey data were entered into an outbreak questionnaire developed using the Notifiable Conditions Information Management System (NCIMS) and analysed using IBM SPSS Statistics version 23.0 [12]. Pertussis cases were matched to cases by age ( \pm 5 years) and location; NBH controls were matched to cases by location (urban or rural). Univariate analysis was carried out to compare cases with each control group separately and an adjusted estimate of the OR and their $95 \%$ confidence intervals (CI) were calculated from matched pairs of cases and controls for various risk factors.

For each case-control group, variables with a significant OR in the univariate analysis were placed into a multivariable regression for each matched group respectively. No potential confounders were identified in the regression model with the Pertussis cases. In the regression model with the NBH controls, age and sex were controlled as potential confounders. A backward stepwise elimination process was employed, using a likelihood ratio test to produce the most parsimonious model [13]. All variables with a Wald $\chi^{2}$ statistically significant at the $P$-value of $<0.05$ were considered significant. OR and $95 \%$ CI for the association were reported. Cases for whom we could not identify suitable matching control subjects were excluded from the matched casecontrol analysis.

\section{Results}

Of the 217 giardiasis cases invited to participate in the study, 68 (31.3\%) consented to be interviewed for the study (see Fig. 1). Letters were mailed to 1983 randomly selected households residing in the same neighbourhood as cases (Fig. 1). Of these, 113 controls (5.7\%) returned a completed questionnaire and were included in the study. A total of 75 Pertussis cases were selected from NCIMS and contacted via telephone. Of these, 36 (48.0\%) agreed to be interviewed for the study and, 26 (34.7\%) could not be contacted after three telephone call attempts. To reduce the risk of selection bias, two separate matched analyses were done: one which combined 21 cases and 36 Pertussis cases and the other matched 68 cases and $68 \mathrm{NBH}$ controls.

\section{Demographic characteristics}

The distribution of the cases and controls by age and gender is presented in Table 1. Cases and controls were similar with regard to language spoken at home, highest level of education and indigenous status. Cases and controls mainly originated from urban areas in SWS as opposed to rural. More than half of case-patients (40 or 58.8\%), compared with 27 (40.3\%) NBH controls and 15 
Table 1. Demographics of cases and controls

\begin{tabular}{|c|c|c|c|c|c|c|}
\hline Demographics & $\begin{array}{c}\text { Cases } \\
\%(N=68)\end{array}$ & $\begin{array}{l}\text { Neighbourhood controls } \\
\qquad \%(N=113)\end{array}$ & $P$-value & $\begin{array}{c}\text { Cases } \\
\%(N=21)\end{array}$ & $\begin{array}{l}\text { Pertussis cases } \\
\qquad(N=36)\end{array}$ & $P$-value \\
\hline Median age in years (range) & $\begin{array}{c}8.0 \text { (Range 0-83; } \\
\text { Mean 18.3 } \\
(13.6-23.0) \pm 19.36)\end{array}$ & $\begin{array}{c}57.5 \text { (Range 0-84; } \\
\quad \text { Mean 51.0 } \\
(46.0-56.1) \pm 20.8)\end{array}$ & $N R^{a}$ & $\begin{array}{c}7.0 \text { (Range 1-70; } \\
\quad \text { Mean 19.4 } \\
(10.0-28.9) \pm 20.8)\end{array}$ & $\begin{array}{c}8.0 \text { (Range 1-65; } \\
\quad \text { Mean 16.3 } \\
(10.2-22.3) \pm 17.9)\end{array}$ & NR \\
\hline $0-4$ years & $36.8 \%(25)$ & $2.9 \%(2)$ & $0.001^{\star}$ & $28.6 \%(6)$ & $41.7 \%(15)$ & 0.242 \\
\hline 5 years or older & $63.2 \%(43)$ & $97.1 \%(66)$ & & $71.4 \%(15)$ & $58.3 \%(21)$ & \\
\hline \multicolumn{7}{|l|}{ Gender } \\
\hline Male & $58.8 \%(40)$ & $39.7 \%(27)$ & $0.027^{\star}$ & $47.6 \%(10)$ & $41.7 \%(15)$ & 0.662 \\
\hline Female & $41.2 \%(28)$ & $60.3 \%(41)$ & & $52.4 \%(11)$ & $58.3 \%(21)$ & \\
\hline \multicolumn{7}{|l|}{ Residence } \\
\hline Urban & $86.8 \%(59)$ & $86.8 \%(59)$ & 1.000 & $76.2 \%(16)$ & $75.0 \%(27)$ & 0.920 \\
\hline Rural & $13.2 \%(9)$ & $13.2 \%(9)$ & & $23.8 \%(5)$ & $25.0 \%(9)$ & \\
\hline \multicolumn{7}{|l|}{ Language spoken at home } \\
\hline English & $86.6 \%(58)$ & $100.0 \%(10)$ & NR & $76.2 \%(16)$ & $100.0 \%(4)$ & NR \\
\hline Arabic & $3.0 \%(2)$ & $0.0 \%(0)$ & & $0.0 \%(0)$ & $0.0 \%(0)$ & \\
\hline Hindi & $3.0 \%(2)$ & $0.0 \%(0)$ & & $9.5 \%(2)$ & $0.0 \%(0)$ & \\
\hline Other languages ${ }^{\mathrm{b}}$ & $7.4 \%(5)$ & $0.0 \%(0)$ & & $14.3 \%(3)$ & $0.0 \%(0)$ & \\
\hline \multicolumn{7}{|l|}{ Aboriginality } \\
\hline Aboriginal but not Torres Strait Islander & $0.0 \%(0)$ & $1.5 \%(1)$ & NR & $0.0 \%(0)$ & $2.8 \%(1)$ & NR \\
\hline Not Aboriginal and Torres Strait Islander & $98.5 \%(67)$ & $83.8 \%(57)$ & & $95.2 \%(20)$ & $97.2 \%(35)$ & \\
\hline Both Aboriginal and Torres Strait Islander & $1.5 \%(1)$ & $0.0 \%(0)$ & & $4.8 \%(1)$ & $0.0 \%(0)$ & \\
\hline \multicolumn{7}{|l|}{ Highest Level of Education ${ }^{c}$} \\
\hline No formal education & $4.4 \%(3)$ & $2.9 \%(2)$ & NR & $4.8 \%(1)$ & $0.0 \%(0)$ & NR \\
\hline Primary or elementary school (Year K-6 or equivalent) & $1.5 \%(1)$ & $4.4 \%(3)$ & & $4.8 \%(1)$ & $2.8 \%(1)$ & \\
\hline Secondary school (Year 7-12 or equivalent) & $22.1 \%(15)$ & $22.1 \%(15)$ & & $9.5 \%(2)$ & $41.7 \%(15)$ & \\
\hline Vocational (e.g. TAFE or skills training) & $27.9 \%(19)$ & $33.8 \%(23)$ & & $28.6 \%(6)$ & $25.0 \%(9)$ & \\
\hline University & $36.8 \%(25)$ & $35.3 \%(24)$ & & $42.9 \%(9)$ & $30.6 \%(11)$ & \\
\hline Other form of education & $7.3 \%(5)$ & $1.5 \%(1)$ & & $9.5 \%(2)$ & $0.0 \%(0)$ & \\
\hline
\end{tabular}

aNR not reported and/or calculated.

Other languages spoken by one person each: Bengali, Cantonese, Macedonian, Mandarin and Spanish.

If the case was under 12 years of age, the educational level was provided by the parent/head of household answering the survey

'Statistically significant $(P<0.05)$. 
(41.7\%) Pertussis cases were males. The age distributions varied between cases and controls with the median age being $8( \pm 19.4)$ years for giardiasis cases, $58( \pm 20.8)$ years for $\mathrm{NBH}$ controls and for Pertussis cases, $8( \pm 17.9)$ years (see Table 1$)$.

In comparison with the cases, there were significantly fewer NBH controls aged $0-4$ years $(36.8 \%$ vs $2.9 \%)$. Conversely, significantly more Pertussis cases were aged $0-4$ years (28.6\% vs $41.7 \%$ ). There were also significantly more older females as NBH controls in comparison with the Pertussis cases which had significantly more children aged $<5$ years.

Univariate analyses revealed that males were significantly more likely to be cases when compared with NBH controls, hence sex was controlled as a potential confounder in the multivariable analysis. When controlling for sex in the multivariable analysis, cases aged under 5 years had a seven times greater risk of Giardia infection $(\mathrm{aOR}=7.08$; 95\% CI 1.02-49.36) when compared with $\mathrm{NBH}$ controls. There was no difference between the ages and genders of giardiasis cases and Pertussis cases.

\section{Risk factors for giardiasis}

Univariate analysis of the comparison between $\mathrm{NBH}$ controls and cases revealed that cases who, (a) were males aged under 5 years, (b) visited their/parent's country of birth, (c) had a child that attends childcare, (d) had a household member diagnosed with a gastrointestinal illness, (e) were individuals who swim in pools, (f) had contact with domestic animals, wildlife or livestock and $(\mathrm{g})$ were individuals who visited a farm, zoo or wildlife park, were at increased risk for giardiasis $(P<0.05)$ (Table 2$)$. Those who temporarily stored their water in jars, bottles or cisterns at home and for those who consumed green salad or lettuce on a daily basis were at a decreased risk $(P<0.05)$ (Table 2$)$. When age and location were controlled in the multivariable analysis, all variables lost their significance except for having a member of household diagnosed with a gastrointestinal illness and having contact with the farm, domestic or wild animals. Those who reported swimming in pools had an elevated risk, but this was not significant $(P=0.06)$ (Table 2$)$.

The univariate analysis matching cases with the second group of controls (i.e. Pertussis cases) found that giardiasis cases were more likely to have travelled overseas and had a household member diagnosed with a gastrointestinal illness. Notably, there was a negative association found between giardiasis cases and living in close proximity to wildlife. All three variables except travelling overseas and outside Australia lost their significance in the multivariable analysis (Table 2).

\section{Discussion}

This matched case-control study represents the value of continuing to monitor giardiasis in south-western Sydney and other parts of NSW and recommends further studies to examine the genotypes in circulation and their potential for zoonotic transmission. The results from this study indicate that some common risk factors of Giardia infection seen in other developed countries were not found to be significant risk factors in south-western Sydney.

Notably, the multivariable analyses among cases and $\mathrm{NBH}$ controls and cases and Pertussis cases found no significant association between giardiasis and those using water sourced from alternative supplies such as roof-harvested rainwater (RHRW), tank water or bore wells. An overall low number of individuals reporting drinking non-municipal water long-term may lead to this lack of association [14]. However, the result is in keeping with other Australian studies that could not identify untreated RHRW tanks as sources of infection for giardiasis, which is likely due to the fact that RHRW tanks are likely to be mainly used for potable replacement for flushing toilets, washing clothes, or watering gardens $[14,15]$.

Furthermore, while initial univariate analyses between cases and NBH controls found a significant association between giardiasis and those who reported swimming in pools (chlorinated, salt-water or non-chlorinated) 3 months prior to illness onset, this significance was lost in the multivariable model that controlled for age and sex. This suggests there may be a relationship between age, sex and swimming that is confounding their association with giardiasis infection in this setting. On the other hand, there are multiple studies that have established the association between swimming in pools and giardiasis infection [16-18].

Giardiasis cases were also more likely to have a household member diagnosed with a gastrointestinal illness when compared with $\mathrm{NBH}$ controls. A similar risk found in the univariate analysis with Pertussis cases, may be due to a low response rate. Notwithstanding, studies in Turkey and other countries have reported an increased risk of infection amongst household members infected with giardiasis $[19,20]$. This indicates a potential for person-to-person transmission of infection occurring within households in SWS with infected family members (or household members) serving as sources of infection. There is also the prospect of transmission through food or water prepared by the infected individual. This study emphasises the importance of screening all household members for giardiasis once a case has been diagnosed.

In this study, the multivariable analysis found a seven times greater risk of infection for those aged under 5 years. However, when compared with Pertussis cases, the risk was insignificant. While other case-control studies have observed no significant risk associated with age, it is more likely that this result is due to the small participant numbers in the Pertussis cases group. Individuals of all age groups can be infected by $G$. duodenalis although the majority of literature maintains that giardiasis is most prevalent in school-age and younger children [21, 22]. Children tend to have a higher exposure to contaminated faeces particularly in close-contact facilities such as childcare centres putting them at greater risk of infection $[16,23,24]$.

While univariate analyses among cases and $\mathrm{NBH}$ controls observed that males were at an increased risk of giardiasis, this association lost its significance in the multivariable analysis after being controlled for sex and age and was likely due to the fact that there were overwhelmingly more females among $\mathrm{NBH}$ controls $[25,26]$.

Cases coming in contact with domestic animals, farm animals and even wildlife were at increased risk of infection when compared with $\mathrm{NBH}$ controls, but not when compared with Pertussis cases. The lack of significance, when compared with the Pertussis cases, may be due to a lack of difference in exposure between the two groups, hence diluting the risk. The possible role of animals as a source of $G$. duodenalis infection to humans is still unclear, although most studies agree that animals can play an indirect role in transmission [6, 27]. Molecular investigations on $G$. duodenalis and the potential for zoonotic transmission observed that humans can only be infected with human-specific assemblages (A or $\mathrm{B}$ ) and not from animal-adapted genotypes $(\mathrm{C}-\mathrm{H})$ [28]. A possible explanation for the present results is that animals are carriers of assemblages $\mathrm{A}$ or $\mathrm{B}$ and act as vehicles 
Table 2. Univariate and multivariable analysis of risk factors for $G$. duodenalis infection

\begin{tabular}{|c|c|c|c|c|c|c|c|c|}
\hline Risk Factors & $\begin{array}{c}\text { Cases } \\
\%(N=68)\end{array}$ & $\begin{array}{l}\text { Neighbourhood Controls } \\
\qquad \%(N=68)\end{array}$ & $\begin{array}{l}\text { Unadjusted OR } \\
\quad(95 \% \mathrm{Cl})\end{array}$ & $\begin{array}{l}\text { Adjusted } O R^{b} \\
(95 \% \mathrm{Cl})\end{array}$ & $\begin{array}{l}\text { Cases } \\
\%(N=21)\end{array}$ & $\begin{array}{c}\text { Pertussis cases } \\
\%(N=36)\end{array}$ & $\begin{array}{l}\text { Unadjusted } \\
\text { OR }(95 \% \mathrm{Cl})\end{array}$ & $\begin{array}{c}\text { Adjusted } \\
\mathrm{OR}^{\mathrm{C}}(95 \% \mathrm{CI})\end{array}$ \\
\hline \multicolumn{9}{|l|}{ Gender } \\
\hline Male & $58.8 \%(40)$ & $40.3 \%(27)$ & $2.17(1.09-4.30)^{\star}$ & $1.31(0.47-3.67)$ & $47.6 \%(10)$ & $41.7 \%(15)$ & $1.27(0.43-3.76)$ & $N R^{d}$ \\
\hline Female & $41.2 \%(28)$ & $60.3 \%(41)$ & & & $52.4 \%(11)$ & $58.3 \%(21)$ & & \\
\hline \multicolumn{9}{|l|}{ Age Category } \\
\hline $0-4$ years & $36.8 \%(25)$ & $2.9 \%(2)$ & $19.19(4.32-85.18)^{\star}$ & $7.08(1.02-49.36)^{\star}$ & $28.6 \%(6)$ & $41.7 \%(15)$ & $0.56(0.18-1.78)$ & NR \\
\hline 5 years or older & $63.2 \%(43)$ & $97.1 \%(66)$ & & & $71.4 \%(15)$ & $58.3 \%(21)$ & & \\
\hline \multicolumn{9}{|l|}{ Travel within Australia } \\
\hline Yes & $8.8 \%(6)$ & $10.8 \%(7)$ & $0.80(0.25-2.53)$ & NR & $9.5 \%(2)$ & $8.3 \%(3)$ & $1.16(0.18-7.56)$ & NR \\
\hline \multicolumn{9}{|l|}{ Travel overseas } \\
\hline Yes & $19.1 \%(13)$ & $11.9 \%(8)$ & $1.74(0.67-4.53)$ & NR & $23.8 \%(5)$ & $2.8 \%(1)$ & $10.94(1.18-101.41)^{\star}$ & $\begin{array}{c}19.89 \\
(2.00-197.37)^{\star}\end{array}$ \\
\hline \multicolumn{9}{|c|}{ Visit country of birth or parent's country of birth } \\
\hline Yes & $76.9 \%(10)$ & $18.8 \%(3)$ & $14.44(2.39-87.40)^{\star}$ & NR & $80.0 \%(4)$ & $0.0 \%(0)$ & NR & NR \\
\hline \multicolumn{9}{|l|}{ Countries visited overseas } \\
\hline South \& Southeast Asia & $38.5 \%(5)$ & $37.5 \%(3)$ & NR & NR & $20.0 \%(1)$ & $100.0 \%(1)$ & NR & NR \\
\hline West Central Asia/North Africa & $7.7 \%(1)$ & $12.5 \%(1)$ & & & $20.0 \%(1)$ & $0.0 \%(0)$ & & \\
\hline Oceania & $30.8 \%(4)$ & $25.0 \%(2)$ & & & $40.0 \%(2)$ & $0.0 \%(0)$ & & \\
\hline Europe & $0.0 \%(0)$ & $12.5 \%(1)$ & & & $0.0 \%(0)$ & $0.0 \%(0)$ & & \\
\hline Latin America \& Caribbean & $7.7 \%(1)$ & $12.5 \%(1)$ & & & $0.0 \%(0)$ & $0.0 \%(0)$ & & \\
\hline Multiple Regions & $15.4 \%(2)$ & $0.0 \%(0)$ & & & $20.0 \%(1)$ & $0.0 \%(0)$ & & \\
\hline \multicolumn{9}{|l|}{ Camp or caravan } \\
\hline Yes & $9.0 \%(6)$ & $10.4 \%(7)$ & $0.84(0.27-2.66)$ & NR & $9.5 \%(2)$ & $0.0 \%(0)$ & NR & NR \\
\hline \multicolumn{9}{|c|}{ Children at home attending childcare } \\
\hline Yes & $50.0 \%(34)$ & $7.7 \%(5)$ & $12.00(4.29-33.57)^{\star}$ & $2.46(0.63-9.70)$ & $42.9 \%(9)$ & $30.6 \%(11)$ & $1.71(0.56-5.21)$ & NR \\
\hline \multicolumn{9}{|c|}{ Member of household diagnosed with a gastrointestinal illness } \\
\hline Yes & $24.2 \%(16)$ & $1.5 \%(1)$ & $21.44(2.75-167.08)^{*}$ & $15.89(1.53-164.60)^{*}$ & $21.1 \%(4)$ & $5.6 \%(2)$ & $4.53(0.75-27.50)^{\star}$ & NR \\
\hline \multicolumn{9}{|l|}{ Unfiltered or non-boiled tap water } \\
\hline Yes & $65.2 \%(43)$ & $73.5 \%(50)$ & NR & NR & $65.0 \%(13)$ & $58.3 \%(21)$ & $1.33(0.43-4.12)$ & NR \\
\hline \multicolumn{9}{|l|}{ Filtered tap water } \\
\hline Yes & $45.5 \%(30)$ & $33.8 \%(22)$ & NR & NR & $40.0 \%(8)$ & $38.9 \%(14)$ & $1.05(0.34-3.20)$ & NR \\
\hline \multicolumn{9}{|l|}{ Sydney water connected to home } \\
\hline Yes & $91.7 \%(55)$ & $86.8 \%(59)$ & $1.68(0.53-5.32)$ & NR & $82.4 \%(14)$ & $91.2 \%(31)$ & $0.45(0.08-2.52)$ & NR \\
\hline
\end{tabular}




\begin{tabular}{|c|c|c|c|c|c|c|c|c|}
\hline Yes & $5.9 \%(4)$ & $13.2 \%(9)$ & $0.41(0.12-1.40)$ & NR & $9.5 \%(2)$ & $2.8 \%(1)$ & $3.68(0.31-43.32)$ & NR \\
\hline \multicolumn{9}{|c|}{ Bore water or shallow well water used in home } \\
\hline Yes & $0.0 \%(0)$ & $1.5 \%(1)$ & NR & NR & $0.0 \%(0)$ & $0.0 \%(0)$ & NR & NR \\
\hline \multicolumn{9}{|c|}{ Tank water used in home } \\
\hline Yes & $22.1 \%(15)$ & $10.3 \%(7)$ & $2.47(0.94-6.50)$ & NR & $19.0 \%(4)$ & $27.8 \%(10)$ & $0.61(0.17-2.27)$ & NR \\
\hline \multicolumn{9}{|c|}{ Temporary storage of water in jars, bottles, cisterns at home } \\
\hline Yes & $1.6 \%(1)$ & $32.4 \%(22)$ & $0.03(0.00-0.26)^{\star}$ & NR & $0.0 \%(0)$ & $2.8 \%(1)$ & NR & NR \\
\hline \multicolumn{9}{|c|}{ Swimming in pool } \\
\hline Yes & $57.6 \%(38)$ & $28.4 \%(19)$ & $3.43(1.67-7.05)^{\star}$ & $2.63(0.95-7.27)$ & $52.4 \%(11)$ & $52.8 \%(19)$ & $0.98(0.34-2.89)$ & NR \\
\hline \multicolumn{9}{|c|}{ Swimming in river, lake, lagoon, pond or similar setting } \\
\hline Yes & $13.2 \%(9)$ & $4.4 \%(3)$ & $3.31(0.85-12.79)$ & NR & $9.5 \%(2)$ & $13.9 \%(5)$ & $0.65(0.12-3.71)$ & NR \\
\hline \multicolumn{9}{|c|}{ Swimming in the ocean } \\
\hline Yes & $10.3 \%(7)$ & $16.2 \%(11)$ & $0.60(0.22-1.64)$ & NR & $14.3 \%(3)$ & $16.7 \%(6)$ & $0.83(0.19-3.75)$ & NR \\
\hline \multicolumn{9}{|c|}{ Always wash hands before eating } \\
\hline Yes & $60.3 \%(41)$ & $67.2 \%(45)$ & $0.74(0.37-1.50)$ & NR & $61.9 \%(13)$ & $69.4 \%(25)$ & $0.72(0.23-2.22)$ & NR \\
\hline \multicolumn{9}{|c|}{ Always wash hands after playing with animals } \\
\hline Yes & $80.3 \%(49)$ & $76.5 \%(52)$ & $1.26(0.54-2.92)$ & NR & $76.5 \%(13)$ & $74.3 \%(26)$ & $1.13(0.29-4.35)$ & NR \\
\hline \multicolumn{9}{|c|}{ Changing nappies of child/children } \\
\hline Yes & $13.8 \%(9)$ & $20.9 \%(14)$ & $0.61(0.24-1.52)$ & NR & $14.3 \%(3)$ & $8.3 \%(3)$ & $1.83(0.34-10.04)$ & NR \\
\hline \multicolumn{9}{|c|}{ Engaging in sexual activity with contact with faeces } \\
\hline Yes & $1.8 \%(1)$ & $1.5 \%(1)$ & $1.20(0.07-19.57)$ & NR & $0.0 \%(0)$ & $0.0 \%(0)$ & NR & NR \\
\hline \multicolumn{9}{|c|}{ Onsite septic system at home } \\
\hline Yes & $12.1 \%(8)$ & $14.9 \%(10)$ & $0.79(0.29-2.14)$ & NR & $15.8 \%(3)$ & $22.9 \%(8)$ & $0.63(0.15-2.74)$ & NR \\
\hline \multicolumn{9}{|c|}{ Contact with farm/domestic animal/wildlife } \\
\hline Yes & $61.8 \%(42)$ & $32.4 \%(22)$ & $3.38(1.67-6.84)^{\star}$ & $3.03(1.08-8.54)^{\star}$ & $71.4 \%(15)$ & $72.2 \%(26)$ & $0.96(0.29-3.18)$ & NR \\
\hline \multicolumn{9}{|c|}{ Visited a farm, zoo, wildlife park } \\
\hline Yes & $28.4 \%(19)$ & $9.1 \%(6)$ & $3.96(1.47-10.69)^{\star}$ & NR & $19.0 \%(4)$ & $38.9 \%(14)$ & $0.37(0.10-1.33)$ & NR \\
\hline \multicolumn{9}{|c|}{ Wildlife in close proximity to house } \\
\hline Yes & $26.5 \%(18)$ & $17.9 \%(12)$ & $1.65(0.72-3.76)$ & NR & $14.3 \%(3)$ & $41.7 \%(15)$ & $0.23(0.06-0.94)^{\star}$ & $\begin{array}{c}0.54 \\
(0.11-2.65)\end{array}$ \\
\hline \multicolumn{9}{|c|}{ Consumes green salad/lettuce daily } \\
\hline Yes & $17.9 \%(12)$ & $38.8 \%(26)$ & $0.34(0.16-0.76)^{\star}$ & $0.48(0.15-1.52)$ & $14.3 \%$ (3) & $22.2 \%(8)$ & $0.58(0.14-2.49)$ & NR \\
\hline
\end{tabular}

*Statistically significant $(\mathrm{P}<0.05)$.

Unadjusted odds ratio.

Odds ratio from multivariable model adjusted for sex and age and all exposures that have been previously reported to be associated with giardiasis and showed a significant association $(P<0.05)$ in the univariate model.

Codds ratio from multivariable model adjusted for sex and all exposures that have been previously reported to be associated with giardiasis and showed a significant association $(P<0.05)$ in the univariate model.

${ }^{\mathrm{d}} \mathrm{NR}$ not reported and/or calculated. 
for mechanical transmission to humans who come in contact with animal's faeces at parks or wildlife settings where hand-washing facilities may not be available [29], or other environmental exposures to cysts attached to the fur of domestic animals [30].

Interestingly, the vast majority $(80.9 \%)$ of $G$. duodenalis cases did not report travelling overseas within the 3 months prior to illness onset suggesting that most of the giardiasis cases were locally acquired. This is the first case-control study to examine travel history amongst giardiasis cases in this setting and is consistent with other case-control studies conducted in other developed countries $[16,23,31]$. However, multivariable analyses found that when compared with Pertussis cases, giardiasis cases were 20 times more likely to have been travelling overseas. The most popular countries visited were in South \& South-East Asia, West Central Asia/North Africa and Oceania. Overseas travel to endemic regions is widely believed to be the principal risk factor for giardiasis in developed countries. However, due to detection bias associated with physicians testing for giardiasis more commonly among returning travellers, overseas acquired infection rate is likely to be overestimated; and consequently underestimating locally acquired giardiasis [32].

There are some limitations to this study. Although care was taken to recruit controls representative of the source population of cases, some selection bias may exist among controls. There was a larger response rate among older females residents in urban areas in SWS, indicating that women were more likely to respond to the $\mathrm{NBH}$ control questionnaire. There was also an underrepresentation of children seen in the NBH controls when compared with Pertussis cases. This selection bias emphasised the sex and age differences between cases and NBH controls and could explain why some exposures were also present among the control group, thus diluting the exposure rates amongst cases. A matched analysis was done to reduce selection bias and improve internal validity, by controlling for the sex, age and region of residence differences between cases and $\mathrm{NBH}$ controls. The matched design reduced the risk of error from the confounding effect of age, sex and location but due to the resulting close matching on these variables, their effects on giardiasis risk could not be assessed. However, controlling for these wellknown confounders was valuable as it allowed the assessment of other risk factors without their confounding influences. Admission risk bias is a potential problem with Pertussis cases, which were selected based on being a group of patients available through NCIMS, did not have gastrointestinal symptoms or diagnosed with giardiasis and hence they may have a different exposure profile to the general population. Since giardiasis cases matched to pertussis cases were quite similar in sex distribution, there was no association and hence no further need for controlling this variable. Like most studies that utilises surveillance data as a sampling frame, only symptomatic $G$. duodenalis cases that sought medical attention and had a positive laboratory test were included in the study. This means that this study represents only a proportion of the overall burden of the disease in the community. Cases with undiagnosed and asymptomatic giardiasis would not have been considered. Therefore, this study cannot be generalised to all of Australia and must be interpreted in the context of these limitations.

\section{Conclusion}

The study showed an increased risk of giardiasis in children aged under 5 years, amongst individuals who have a household member diagnosed with a gastrointestinal illness and have contact with domestic animals, wildlife or livestock. The study also found that cases who travelled overseas were at a greater risk of infection. There is a need to educate residents living in urban areas in SWS on the potential of person-to-person transmission of giardiasis; particularly if a household member is ill with gastroenteritis. Targeted intervention and health messages are needed for the parents/carers of younger children especially during high-risk seasons such as warmer months, with emphasis on potential risks and appropriate hygiene practices when visiting farms and wildlife parks or where contact with animals is to be expected. Likewise, people travelling overseas to endemic countries should be appropriately informed of the risks and possible control strategies that can be implemented. This study illustrates the value of continuing to monitor giardiasis in south-western Sydney and other parts of NSW and recommends further studies to examine the genotypes in circulation and their potential for zoonotic transmission.

Supplementary material. The supplementary material for this article can be found at https://doi.org/10.1017/S0950268818002637.

Acknowledgments. The authors thank the staff at SWSLHD Public Health Unit for their support in recruiting survey participants and accessing the surveillance data. They also thank all who participated in piloting the questionnaire. The valuable contributions of two anonymous referees are acknowledged.

Conflict of interest. None.

Ethical standards. Ethical approval for this study was received from the South-Western Sydney Local Health District Human Research Ethics Committee which is accredited by the NSW Ministry of Health (HREC approval number: HE16/079 LNR).

\section{References}

1. Esch KJ and Petersen CA (2013) Transmission and epidemiology of zoonotic protozoal diseases of companion animals. Clinical Microbiology Reviews 26, 58-85.

2. Wensaas KA et al. (2012) Irritable bowel syndrome and chronic fatigue 3 years after acute giardiasis: historic cohort study. Gut 61, 214-219.

3. MAL-ED Network Investigators (2014) The MAL-ED study: a multinational and multidisciplinary approach to understand the relationship between enteric pathogens, malnutrition, gut physiology, physical growth, cognitive development, and immune responses in infants and children up to 2 years of age. Clinical Infectious Diseases 59(Suppl. 4), 193-206.

4. Savioli L, Smith H and Thompson A (2006) Giardia and cryptosporidium join the 'neglected diseases initiative'. Trends in Parasitology 22, 203-208.

5. NSW Government Health (2017) Communicable diseases, "Giardiasis control guideline". Available at http://www.health.nsw.gov.au/Infectious/ controlguideline/Pages/giardiasis.aspx (Accessed 20 February 2017).

6. Fletcher SM et al. (2012) Enteric protozoa in the developed world: a public health perspective. Clinical Microbiology Review 25, 420-449.

7. Communicable Diseases Branch (2017) Giardiasis Notifications in NSW Residents, by Year of Disease Onset. January 2014 to December 2014, Sydney, New South Wales: Ministry of Health.

8. Fletcher SM et al. (2015) Descriptive epidemiology of infectious gastrointestinal illnesses in Sydney, Australia, 2007-2010. Western Pacific Surveillance and Response Journal 6, 7-16.

9. Yang $\mathbf{R}$ et al. (2010) High prevalence Giardia duodenalis assemblage B and potentially zoonotic subtypes in sporadic human cases in Western Australia. International Journal for Parasitology 40, 293-297.

10. Meloni BP et al. (1993) The prevalence of Giardia and other intestinal parasites in children, dogs and cats from aboriginal communities in the Kimberley. The Medical Journal of Australia 3, 157-159. 
11. Center for Surveillance, Epidemiology \& Laboratory Services (CSELS) Epi Info ${ }^{\mathrm{TM}}$ Companion App for iOS v3.3.3. Atlanta, GA; USA, 2011.

12. IBM SPSS Corporation (2015) PASW Statistics. Chicago, IL: SPSS Inc. an IBM Company.

13. Vandekerckhove J, Matzke D, Wagenmakers EJ (2014) Model comparison and the principle of parsimony. In Busemeyer JR, Wang Z, Townsend JT and Eidels A (eds), The Oxford Handbook of Computational and Mathematical Psychology. New York, NY: Oxford University Press, pp. 1-29.

14. Heyworth J et al. (2006) Consumption of untreated tank rainwater and gastroenteritis among young children in South Australia. International Journal of Epidemiology 35, 1051-1058.

15. Tan $\mathbf{H}$ et al. (2006) Identifying the presence of cryptosporidium, Giardia, Campylobacter and Salmonella spp. in private rainwater supplies. Environmental Health 6, 53-59.

16. Minetti C et al. (2015) Case-control study of risk factors for sporadic giardiasis and parasite assemblages in north west England. Journal of clinical microbiology 53, 3133-3140.

17. Painter JE et al. (2015) Giardiasis surveillance - United States, 20112012. Morbidity and Mortality Weekly report Surveillance summaries. Morbidity and Mortality Weekly Report (MMWR) 64, 15-25.

18. Hoque ME et al. (2002) Risk of giardiasis in Aucklanders: a case-control study. International Journal of Infectious Diseases 6, 191-197.

19. Balcioglu IC et al. (2003) Incidence of giardiasis among siblings in Turkey. Pediatrics International 45, 311-313.

20. Anuar TS et al. (2012) Giardiasis among different tribes of Orang Asli in Malaysia: highlighting the presence of other family members infected with Giardia intestinalis as a main risk factor. International Journal of Parasitology 42, 871-880.

21. Fletcher SM et al. (2014) Epidemiology and geographical distribution of enteric protozoan infections in Sydney, Australia. Journal of Public Health Research 3, 83-91.
22. Siwila J and Olsen A (2015) Risk factors for infection with soil transmitted helminths, Cryptosporidium spp., and Giardia duodenalis in children enrolled in preschools in Kafue District, Zambia. Epidemiology Research International 7, 1-7.

23. Espelage W et al. (2010) Characteristics and risk factors for symptomatic Giardia lamblia infections in Germany. BMC Public Health 10, 40.

24. Faustini A et al. (2006) The impact of the Catholic Jubilee in 2000 on infectious diseases. A case-control study of giardiasis, Rome, Italy 20002001. Epidemiology and Infection 134, 649-658.

25. Mezei G and Kheifets $\mathbf{L}$ (2006) Selection bias and its implications for case-control studies: a case study of magnetic field exposure and childhood leukaemia. International Journal of Epidemiology 35, 397-406.

26. Geneletti S, Richardson S and Best N (2009) Adjusting for selection bias in retrospective, case-control studies. Biostatistics (Oxford, England) 10, 17-31.

27. Thompson RCA and Smith A (2011) Zoonotic enteric protozoa. Veterinary Parasitology 182, 70-78.

28. Li F et al. (2016) Prevalence and molecular characterization of Cryptosporidium spp. and Giardia duodenalis in dairy cattle in Beijing, China. Veterinary Parasitology 219, 61-65.

29. Choy SH et al. (2014) Prevalence and associated risk factors of Giardia infection among indigenous communities in Rural Malaysia. Scientific Reports 4, 6909.

30. Ballweber LR et al. (2010) Giardiasis in dogs and cats: update on epidemiology and public health significance. Trends in Parasitology 26, 180-189.

31. Reses HE et al. (2018) Risk factors for sporadic Giardia infection in the USA: a case-control study in Colorado and Minnesota. Epidemiology and Infection 146, 1071-1078.

32. Gaut S et al. (2008) Occurrence of Cryptosporidium oocysts and Giardia cysts in Norwegian groundwater wells in bedrock. Journal of Water \& Health 6, 383-388. 